\title{
Automated Self-management (ASM) vs. ASM-Enhanced Collaborative Care for Chronic Pain and Mood Symptoms: the CAMMPS Randomized Clinical Trial
}

\author{
Kurt Kroenke, $M D^{1,2,3}$, Fitsum Baye, $M S^{4}$, Spencer G. Lourens, PhD ${ }^{4}$, Erica Evans ${ }^{1}$, \\ Sharon Weitlauf, $R N^{7}$, Stephanie McCalley ${ }^{7}$, Brian Porter, BS ${ }^{7}$, \\ Marianne S. Matthias, $P h D^{1,2,5}$, and Matthew J. Bair, MD ${ }^{1,2,3}$
}

\begin{abstract}
'VA HSR\&D Center for Health Information and Communication, Roudebush VA Medical Center, Indianapolis, IN, USA; ${ }^{2}$ Department of Medicine, Indiana University School of Medicine, Indianapolis, IN, USA; ${ }^{3}$ Regenstrief Institute, Inc., Indianapolis, IN, USA; ${ }^{4}$ Department of Biostatistics, Fairbanks School of Public Health, Indianapolis, IN, USA; ${ }^{5}$ Department of Communication Studies, Indianapolis University-Purdue University at Indianapolis, Indianapolis, IN, USA.
\end{abstract}

BACKGROUND: Chronic musculoskeletal pain is often accompanied by depression or anxiety wherein cooccurring pain and mood symptoms can be more difficult to treat than either alone. However, few clinical trials have examined interventions that simultaneously target both pain and mood conditions.

OBJECTIVE: To determine the comparative effectiveness of automated self-management (ASM) vs. ASM-enhanced collaborative care.

DESIGN: Randomized clinical trial conducted in six primary care clinics in a VA medical center.

PARTICIPANTS: Two hundred ninety-four patients with chronic musculoskeletal pain of at least moderate intensity and clinically significant depressive and/or anxiety symptoms.

INTERVENTION: ASM consisted of automated monitoring and 9 web-based self-management modules. Comprehensive symptom management (CSM) combined ASM with collaborative care management by a nurse-physician team. Both interventions were delivered for 12 months.

MAIN MEASURES: Primary outcome was a composite pain-anxiety-depression (PAD) $z$-score consisting of the mean of the BPI, PHQ-9, and GAD-7 z-scores: 0.2, 0.5, and 0.8 represent potentially small, moderate, and large clinical differences. Secondary outcomes included global improvement, health-related quality of life, treatment satisfaction, and health services use.

KEY RESULTS: Both CSM and ASM groups had moderate PAD score improvement at 12 months $(z=-0.65$ and - 0.52, respectively). Compared to the ASM group, the CSM group had a $-0.23(95 \%$ CI, -0.38 to -0.08 ; overall $P=.003$ ) greater decline in composite PAD $z$-score over 12 months. CSM patients were also more likely to report global improvement and less likely to report worsening at $6(P=.004)$ and 12 months $(P=.013)$.

CONCLUSIONS: Two intervention models relying heavily on telecare delivery but differing in resource intensity both

Electronic supplementary material The online version of this article (https://doi.org/10.1007/s11606-019-05121-4) contains supplementary material, which is available to authorized users.

Received September 17, 2018

Revised January 9, 2019

Accepted April 25, 2019

Published online June 21, 2019 produced moderate improvements in pain and mood symptoms. However, the model combining collaborative care led by a nurse-physician team with web-based selfmanagement was superior to self-management alone.

TRIAL REGISTRATION: ClinicalTrials.gov: NCT0175730

KEY WORDS: pain; depression; anxiety; collaborative care; telemedicine; clinical trial.

J Gen Intern Med 34(9):1806-14

DOI: $10.1007 / \mathrm{s} 11606-019-05121-4$

(C) Society of General Internal Medicine 2019

$\mathrm{P}$ ain is the most common physical symptom reported in both the general population and primary care, and musculoskeletal pain is consistently the most common, disabling, and costly of all pain complaints. ${ }^{1,2}$ Depression and anxiety are the two most common mental health problems. ${ }^{1,3}$ Musculoskeletal pain, depressive, and anxiety disorders, termed PAD when they co-occur, account for 6 of the 9 leading causes of disability in the USA. ${ }^{4}$ Pain-anxiety-depression (PAD) can produce a vicious cycle in which the presence of one symptom, if untreated, may negatively affect the response to treatment of the other two symptoms. ${ }^{1}$

Collaborative care interventions involving a centralized care manager-physician specialist team working in partnership with primary care clinicians have proven effective for both depression and pain. ${ }^{3,5-8}$ These interventions typically predominantly use telephone rather than face-to-face care manager visits. The addition of automated management has the potential to further improve efficiency and has also proven effective (and possibly cost-effective) for pain and potentially depression. ${ }^{9-13}$ Concerns remain, however, about the adequacy of automated management for complex patients with pain combined with mental health disorders.

This paper reports the main outcomes of the Comprehensive vs. Assisted Management of Mood and Pain Symptoms (CAMMPS) randomized comparative 
effectiveness trial aimed at testing alternative interventions for patients with PAD. CAMMPS compared automated self-management (ASM) alone to ASM augmented by comprehensive symptom management (CSM). In CSM, ASM is enhanced by collaborative care delivered by a centralized nurse-physician team with a focus on optimized medication management, facilitated mental health care, and collaboration with the study participants' primary care teams.

\section{METHODS}

\section{Study Participants}

Participants were recruited from January 2014 through June 2016. Eligible patients were 18 and older with pain plus psychiatric comorbidity who were receiving care from one of 6 primary care clinics at a large VA Medical Center in the Midwest. Details of the trial protocol have been previously described. ${ }^{1}$

Pain had to be (1) musculoskeletal, either localized (in the arms, legs, back, or neck) or widespread (fibromyalgia); (2) persistent for $\geq 3$ months despite a trial of at least one analgesic medication; and (3) at least moderate in severity, defined as a Brief Pain Inventory average severity score $\geq 5$ in the past week or having at least moderately interfered with work or other activities in the past month.

Psychiatric comorbidity had to meet any 1 of the following 3 criteria: (1) depression of at least moderate severity, defined as a PHQ-8 score $\geq 10$ with either depressed mood and/or anhedonia being endorsed; ${ }^{14}$ (2) anxiety of at least moderate severity, defined as a GAD-7 score $\geq 10 ;{ }^{14}$ (3) mixed anxietydepression defined as a combined PHQ- 8 and GAD-7 score $\geq$ 12. Scores of 6 or higher on each scale represent at least mild depression or anxiety symptoms, respectively, ${ }^{14}$ and this level of symptoms has been shown to be responsive to treatment. ${ }^{8}$

Excluded were individuals who (a) did not speak English; (b) had moderately severe cognitive impairment; (c) had schizophrenia, bipolar disorder, or other psychosis; (d) had a severe or complex mental illness or exhibited suicidal thoughts as their condition is unsuitable for a predominantly telecare intervention; (e) were pregnant; and (f) had an anticipated life expectancy of less than 12 months.

Electronic medical records were used to create a master list of individuals who, within the preceding 36 months, had received an ICD diagnosis of a musculoskeletal pain condition and either a depressive or anxiety disorder. A letter describing CAMMPS was mailed to patients on this list followed by a telephone contact to interested patients to assess study eligibility. Eligible patients were scheduled for an initial study visit where, after providing written informed consent, they underwent a baseline study interview. The trial was approved by the Indiana University institutional review board and the Roudebush VAMC research review committee.

\section{Randomization}

Following the baseline interview, the project coordinator randomized participants to ensure that the research assistants responsible for outcome assessments were blinded to treatment arm assignment. To maintain allocation concealment, assignment to treatment arm was determined by a computer-generated randomization list with randomly varying block sizes of 4 and 8 .

\section{Outcome Measures}

Assessments were conducted at baseline, 1, 3, 6, and 12 months by a research assistant blinded to study group. The primary outcome measure was a composite pain-anxiety-depression (PAD) $z$-score of 3 valid, reliable, and responsive measures addressing each of the symptom targets of our intervention: Brief Pain Inventory (BPI), GAD-7 anxiety scale, and PHQ-9 depression scale. ${ }^{14-16}$ The $z$-score is the individual's scale score minus the sample mean at baseline divided by the baseline sample standard deviation. ${ }^{17}$ The composite PAD $z$-score is the average of the $z$-scores for the 3 scales. The change in $z$ score over time represents the number of standard deviations by which an individual's PAD symptoms improve or worsen over the baseline sample mean. Because the 3 scales have different response options and score ranges, conversion to $z$-scores allowed us to standardize scores across the 3 scales and derive a composite score. The change in $z$-score is equivalent to an effect size for which $0.2,0.5$, and 0.8 represent potential small, moderate, and large clinical changes, respectively. ${ }^{18}$

Two secondary PAD outcome measures were assessed. One was a composite PAD score based on the 8-item scales for pain, anxiety, and depression from the PROMIS-57 Profile. ${ }^{19}$ The second was a global PAD response score based on two items assessing clinical response for pain and for mood symptoms using a 7-item scale (much better, moderately better, a little better, same, a little worse, moderately worse, much worse). ${ }^{1}$ Each global response item was collapsed into worse, same, or better $(0,1$, or 2$)$. Collapsed responses were summed to produce an overall score of $0-1,2,3$, or 4 , indicating that the patient felt worse, the same, partially improved, or much improved.

Additional demographic and secondary psychological, health-related quality-of-life, and disability outcomes are summarized in Table 1 and described in detail elsewhere. ${ }^{1}$ Data on analgesic and psychotropic medications dispensed and health care use over the 12 months of the trial were extracted for each patient from the electronic medical records. Co-interventions (i.e., non-study pain- and mood-specific treatments received during the trial) were elicited by patient report at the 6 - and 12 month outcome assessments.

\section{Automated Self-management Intervention}

ASM consisted of automated symptom monitoring and prompted use of pain and mood self-management modules. Study participants completed regular symptom surveys either by interactive voice recorded (IVR) telephone calls or Internet 
Table 1 Baseline Characteristics of 294 Subjects Enrolled in the CAMMPS Study

\begin{tabular}{|c|c|c|c|c|}
\hline \multirow{2}{*}{$\begin{array}{l}\text { Characteristic } \\
\text { Demographic, no. (\%) }\end{array}$} & \multicolumn{2}{|c|}{ CSM group $(n=147)$} & \multicolumn{2}{|c|}{ ASM group $(n=147)$} \\
\hline & & & & \\
\hline Age, mean (SD), years & 56.9 & $(12.5)$ & 57.9 & $(11.7)$ \\
\hline Male & 124 & $(84.4)$ & 133 & $(90.5)$ \\
\hline White race & 116 & (78.9) & 117 & (79.6) \\
\hline Married & 86 & $(58.5)$ & 81 & $(55.1)$ \\
\hline Education $>$ high school & 107 & $(72.8)$ & 111 & $(74.5)$ \\
\hline Income adequate by self-report & 113 & (76.9) & 121 & $(82.3)$ \\
\hline Employed or retired & 83 & $(56.5)$ & 99 & $(67.3)$ \\
\hline Major depression, probable, no. $(\%)$ & 82 & $(55.8)$ & 80 & $(54.4)$ \\
\hline Posttraumatic stress disorder, probable, no. $(\%) *$ & 73 & $(49.7)$ & 90 & $(61.2)$ \\
\hline Substance use risk intermediate to high, no. (\%) & 81 & $(55.1)$ & 69 & (46.9) \\
\hline Comorbid medical diseases, mean (SD), no. & 2.3 & (1.4) & 2.3 & $(1.4)$ \\
\hline \multicolumn{5}{|l|}{ PAD scale scores [range], mean (SD) } \\
\hline BPI pain total (range, $0-10$ [worst]) & 6.1 & $(2.0)$ & 6.0 & $(1.9)$ \\
\hline PHQ-9 depression (range, $0-27$ [worst]) & 14.4 & $(5.1)$ & 13.7 & $(5.3)$ \\
\hline GAD-7 anxiety (range, $0-21$ [worst]) & 11.3 & $(5.0)$ & 11.1 & $(5.3)$ \\
\hline \multicolumn{5}{|l|}{ Psychological scale scores [range], mean (SD) } \\
\hline SF-36 mental health (range, $0-100$ [best]) & 51.9 & $(19.6)$ & 52.7 & (19.8) \\
\hline PTSD Checklist (range, 17-85 [worst]) & 44.1 & (14.7) & 45.5 & $(15.4)$ \\
\hline PHQ-14 somatization (range, $0-28$ [worst]) & 13.9 & (3.9) & 13.2 & $(4.2)$ \\
\hline AUDIT-C alcohol use (range, $0-12$ [worst]) & 2.2 & $(2.0)$ & 2.7 & $(2.7)$ \\
\hline \multicolumn{5}{|l|}{ Health-related quality of life scores, mean (SD) } \\
\hline \multicolumn{5}{|l|}{ SF-12 (norm $=50$, lower is worse $)$} \\
\hline Physical component summary & 33.2 & $(8.6)$ & 33.2 & $(8.4)$ \\
\hline Mental component summary & 37.6 & $(11.2)$ & 38.2 & $(10.1)$ \\
\hline \multicolumn{5}{|l|}{ SF-36 (range, $0-100$ [best]) } \\
\hline General health perceptions & 37.9 & $(27.0)$ & 37.8 & $(29.0)$ \\
\hline Social functioning & 43.3 & $(26.1)$ & 43.6 & (26.6) \\
\hline Vitality & 27.6 & $(17.0)$ & 27.5 & $(20.4)$ \\
\hline \multicolumn{5}{|l|}{ PROMIS [norm $=50$, higher is worse] } \\
\hline Fatigue & 63.6 & $(8.2)$ & 62.9 & $(8.3)$ \\
\hline Sleep & 59.6 & (7.6) & 59.7 & $(7.8)$ \\
\hline \multicolumn{5}{|l|}{ Disability scores (range), mean (SD) } \\
\hline Sheehan Disability Index (range, $0-10$ [worst]) & 5.7 & $(2.3)$ & 5.7 & $(2.4)$ \\
\hline Health-related disability days in past 4 weeks & 15.7 & $(9.1)$ & 15.2 & $(9.7)$ \\
\hline Percent work effectiveness & 60.1 & $(25.2)$ & 61.6 & $(28.0)$ \\
\hline
\end{tabular}

$* P=.047$. There were no other significant differences between intervention and usual care group

(based upon patient preference). The monitoring schedule was weekly for the first month, twice a month for months $2-6$, and monthly for months $7-12$. The core survey included 7 items assessing pain, anxiety, and depression; 5 items assessing sleep, fatigue, irritability, global change, and symptomrelated impairment; and 3 items to prompt use of the pain and mood self-management modules.

The 12-h web-based self-management program was divided into 9 modules (coping with pain; pain medications; communicating with providers; depression; anxiety; sleep; anger management; cognitive strategies; and problem-solving). For participants without Internet access, binders with the program materials were provided. These topics were partly derived from the nurse-administered self-management program in previous trials ${ }^{6,7}$ and linked to several public domain VA and non-VA websites with interactive self-management programs.

\section{Comprehensive Symptom Management Intervention}

This arm received the ASM intervention plus the addition of collaborative care management by a nurse-physician team. The ASM survey was augmented by additional items that could generate e-mail alerts to the nurse for medication side effects or non-adherence, patient requests for treatment change or a nurse call, or missed automated reports. Also, ASM responses were tabulated in a trend report on a secure website that could be regularly reviewed by the nurse. The nurse had scheduled telephone contacts with the patient at baseline, 1, 4, and 12 weeks, as well as prompted contacts based upon automated monitoring trend reports or e-mail alerts. The nurse and supervising physician (a primary care physician [KK] with expertise in pain and mental disorders) met weekly to discuss new patients as well as CSM patients not responding to treatment. The nurse was hired by the study to cover all primary care sites and coordinated communication with CSM patients, primary care teams, and mental health specialists.

For mood symptoms, patients were offered the option of psychotropic medications, referral to a psychologist embedded in primary care, or combined therapy. Those who preferred medication were offered a referral to psychiatry, but those who refused could receive a psychotropic prescription from their primary care physician. These typically were a selective serotonin reuptake inhibitor (SSRI), bupropion, mirtazapine, venlafaxine, or duloxetine. Suicidal ideation identified by the nurse care manager or research assistants was evaluated and triaged using an algorithm tested in previous trials. ${ }^{20}$ 
For pain symptoms, the nurse-physician team developed a treatment plan based upon evidence-based medication algorithms adapted from previous trials. ${ }^{7,8,21}$ Described in detail elsewhere, ${ }^{1}$ the stepped care analgesic algorithm included (1) acetaminophen or a nonsteroidal anti-inflammatory drug; (2) tricyclic antidepressants; (3) muscle relaxants; (4) gabapentinoids (gabapentin, pregabalin), or serotonin-norepinephrine reuptake inhibitors (duloxetine, venlafaxine). Topical analgesics were considered for areas of localized pain. Opioid analgesics were not tapered or discontinued by the study team for patients taking them on enrollment but were not part of the study analgesic algorithm.

Three criteria, assessed by automated symptom monitoring and during nurse calls, prompted adjustments in treatment: less than a $30 \%$ improvement in PAD symptoms, failure to report global improvement as at least moderate or better, or a patient's desire for a change in treatment. ${ }^{1}$

\section{Statistical Analysis}

Sample size was determined to ensure adequate power for the primary hypothesis that CSM is superior to ASM in reducing composite PAD symptom severity. In previous trials, the average intervention effect size, compared to a usual care control group, was 0.55 for pain and 0.54 for depression. ${ }^{6,}{ }^{22}$ Since CAMMPS compared CSM to an active comparator ASM group, we estimated a smaller effect size of 0.35 , which is half way between a small $(0.2)$ and moderate $(0.5)$ effect size. ${ }^{18} \mathrm{~A}$ between-group composite $z$-score difference of 0.35 approximates an effect size of 0.35 . A sample of 286 patients provided $80 \%$ power to detect a between-group effect size difference of 0.35 using an alpha of .05 and allowing for $10 \%$ attrition.

Groups were compared at baseline using ANOVA for continuous variables and chi-square for categorical variables. Between-group differences in continuous outcomes over the 12 months of the trial were compared using mixed effects model repeated measures (MMRM) analysis, adjusting for baseline value of the outcome and time. ${ }^{8,23}$ Age was the only variable not satisfying the missing-at-random assumption and thus was added to the model to specify that missingness was covariate-dependent. ${ }^{24} \mathrm{~A}$ random intercept was used to adjust for within-subject correlation. Time-specific $(1,3,6$, and 12 months) between-group differences were also compared. Dichotomous variables were analyzed using a repeated measures logistic model. Health care and medication use data were analyzed using negative binomial regression. Because of multiple hypothesis-testing, between-group comparisons for secondary outcomes should be interpreted cautiously unless highly significant $(P<.001)$. The effect size is the between-group difference divided by the pooled baseline standard deviation. Exploratory moderator analyses were conducted by examining treatment group by moderator interactions. All analyses were intention-to-treat according to randomized group and used SAS version 9.3.

\section{RESULTS}

\section{Study Participants}

Figure 1 summarizes the participant flow in CAMMPS. Of the 397 patients determined to be eligible, 294 (74\%) enrolled in the trial. Follow-up rates were high, with outcome assessments completed by $94 \%$ of participants at 1 month, $88 \%$ at 3 months, $86 \%$ at 6 months, and $84 \%$ at 12 months. Because 14 participants had no assessments after baseline, 280 were included in the final analyses. Randomization resulted in CSM $(n=147)$ and ASM $(n=147)$ groups balanced in terms of baseline characteristics (Table 1).

Overall, the sample had a mean age of 57.4 years; $87.4 \%$ were men and $79.3 \%$ were white. Probable major depressive disorder and probable PTSD were met by $55.1 \%$ and $55.4 \%$ of study participants (using the PHQ-9 diagnostic algorithm and PTSD Checklist score $\geq 41$, respectively ${ }^{1}$ ). Pain, anxiety, and depression scores were moderate in severity. The duration of pain was $>10$ years in $57.1 \%(n=168)$, and the median number of painful bodily sites (from a checklist of 17 sites) was 5 . Patients reported needing to cut down on their usual activities for one-half day or more on 15.4 days in the past 4 weeks. Multiple domains of health-related quality-of-life were also substantially diminished.

\section{Pain-Anxiety-Depression Outcomes}

By 12 months, the mean composite PAD $z$-score improved in both the CSM and ASM groups: 0.65 and 0.52 , respectively, representing a moderate effect size (Table 2). The betweengroup difference over 12 months estimated from the MMRM model (which adjusted for baseline score, time, and age) was -0.23 (95\% CI, -0.38 to -0.08 , overall $P=.003$ ). Improvement in the composite PROMIS PAD score and global responder analysis (Fig. 2) also favored the CSM group. Notably, at 12 months, CSM patients were more likely to report being much improved (39.5\% vs. $26.8 \%)$ and less likely to report worsening ( $19.4 \%$ vs. $35.8 \%)$.

Of 8 covariates examined - age, sex, race, medical comorbidity, alcohol use, substance use risk, disability score, and socioeconomic disadvantage (SED) index (1 point each for education < high school, income "not adequate to make ends meet", and unemployment) - only the SED index was a moderator (online Appendix, eTable 1). The overall 12-month CSM treatment effect declined incrementally with $0,1,2$, and 3 SED factors from -.48 to -.30 to -.12 to -.07 , respectively.

\section{Health-Related Quality of Life (HRQOL) Outcomes}

Table 3 summarizes between-group differences for secondary health-related quality of life (HRQOL) outcomes. Depression exhibited a more significant between-group difference $(P=.001)$ than pain $(P=.05)$ or anxiety $(P=.06)$. Outcomes for somatization $(P=.03)$, social functioning $(P=.01)$, vitality $(P=.03)$, and the Sheehan Disability Index $(P=.04)$ also favored CSM. 


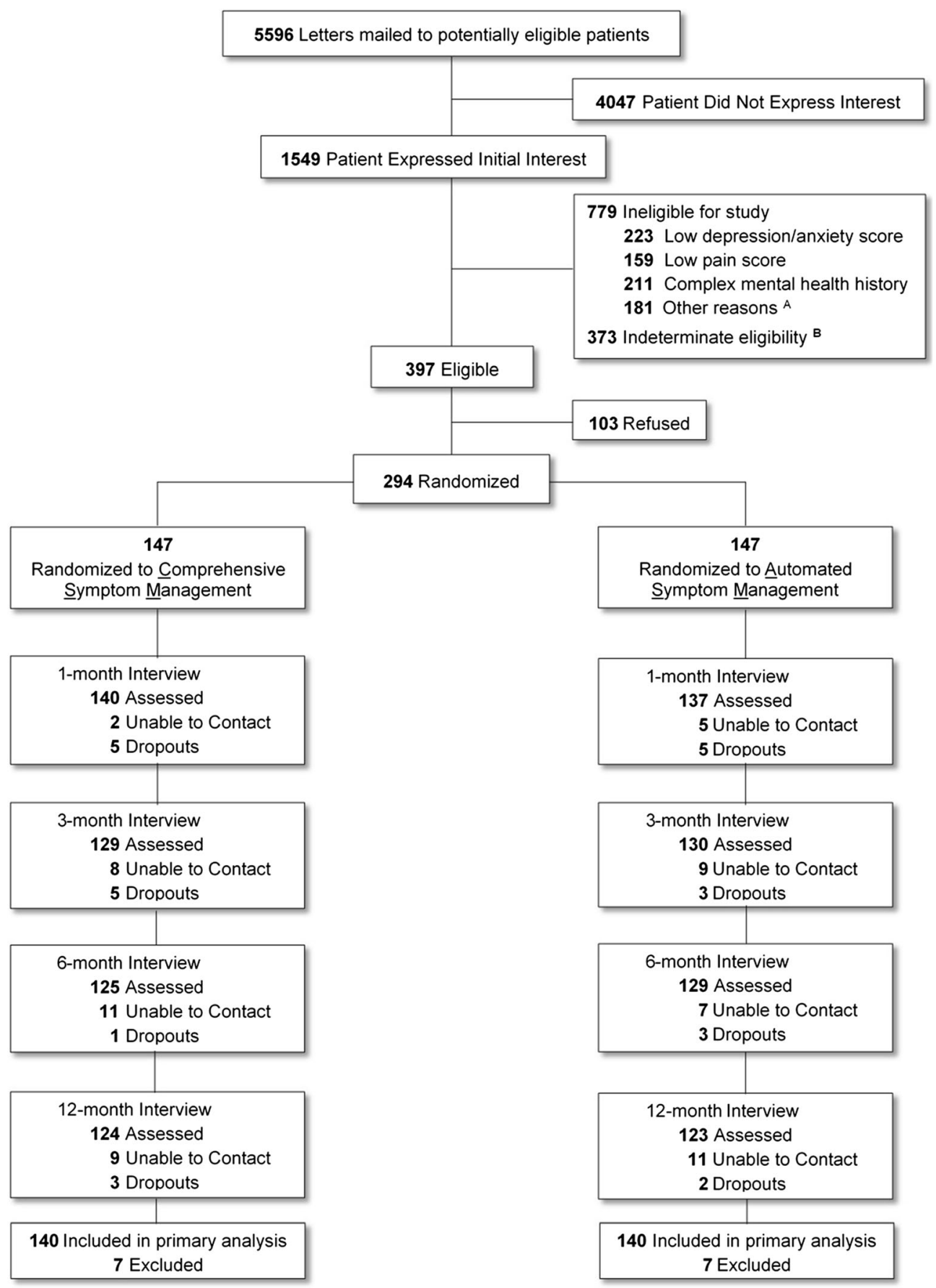

Fig. 1 Flowchart of participants in the CAMMPS trial. Fourteen patients ( 7 in each group) had no follow-up assessments after baseline and were therefore not included in the primary MMRM analysis since at least one follow-up data point is required for MMRM. (A) Other reasons included living out of state $(n=43)$, life expectancy less than 12 months $(n=31)$, no VA primary care physician $(n=29)$, deceased $(n=23)$, cognitive impairment $(n=20)$, severe visual or hearing impairment $(n=12)$, pain less than 3 months $(n=9)$, no analgesics tried $(n=6)$, and other $(n=8)$. (B) Indeterminate eligibility included 245 individuals who refused to do an eligibility interview and 128 who could not be contacted.

\section{Medication and Health Care Use}

Treatment groups were similar at baseline in terms of analgesic and psychotropic use (online Appendix, eTable 2). During the 12-month trial, CSM patients received more discrete analgesics as well as analgesics from more classes $(P<.001$ and $P=.004$, respectively). Psychotropic medication use, mental health visits, and health care use over 12 months was similar in both groups. Also, pain and mood co-interventions did not differ between groups (online Appendix, eTable 3).

The proportion of patients receiving opioids declined in the overall sample from $21.4 \%$ at baseline to $17.0 \%$ by 12 months and was similar between groups. In patients on opioids, doses were stable over the trial (median morphine-equivalent daily dose of $54 \mathrm{mg}$ at both baseline and 12 months). Moreover, 
Table 2 Composite Pain-Anxiety-Depression (PAD) Outcomes in CAMMPS Trial Participants

\begin{tabular}{|c|c|c|c|c|c|}
\hline$\overline{\text { Clinical outcome }}$ & $\begin{array}{l}\text { CSM group } \\
(147)^{\mathrm{a}}\end{array}$ & $\begin{array}{l}\text { ASM group } \\
(147)^{\mathrm{a}}\end{array}$ & Measure (95\% CI) & $\begin{array}{l}\text { Model estimate } \\
(95 \% \text { CI })^{b}\end{array}$ & $\begin{array}{l}\text { Overall } P \\
\text { value }^{\mathrm{b}}\end{array}$ \\
\hline \multicolumn{6}{|l|}{ Primary pain-anxiety-depression (PAD) } \\
\hline Composite PAD z-score, mean (SD) & & & $\begin{array}{l}\text { Time-specific between-group } \\
\text { difference }\end{array}$ & & \\
\hline 1-month follow-up & $-0.40(0.89)$ & $-0.31(0.97)$ & $-0.10(-0.32,0.12)$ & $-0.23(-0.38,-0.08)$ & .003 \\
\hline 3-month follow-up & $-0.51(1.04)$ & $-0.37(0.98)$ & $-0.13(-0.38,0.12)$ & & \\
\hline 6-month follow-up & $-0.64(1.07)$ & $-0.49(1.01)$ & $-0.15(-0.41,0.11)$ & & \\
\hline 12-month follow-up & $-0.65(0.94)$ & $-0.52(1.05)$ & $-0.13(-0.38,0.12)$ & & \\
\hline \multicolumn{6}{|l|}{ Secondary PAD outcomes } \\
\hline $\begin{array}{l}\text { Composite PROMIS PAD T-score, } \\
\text { mean (SD) }\end{array}$ & & & $\begin{array}{l}\text { Time-specific between-group } \\
\text { difference }\end{array}$ & & \\
\hline Baseline & $61.5(6.0)$ & $61.1(6.7)$ & $0.4(-1.1,1.8)$ & $-1.43(-2.54,-0.33)$ & .01 \\
\hline 3-month follow-up & $58.7(7.5)$ & $59.3(7.3)$ & $-0.7(-2.5,1.2)$ & & \\
\hline 6-month follow-up & $57.4(7.6)$ & $58.9(7.5)$ & $-1.5(-3.4,0.3)$ & & \\
\hline 12-month follow-up & $57.6(7.2)$ & $58.8(7.8)$ & $-1.2(-3.1,0.7)$ & & \\
\hline PAD global responder, $N(\%)^{\mathrm{c}}$ & & & Relative risk & $1.72(1.17,2.54)$ & .007 \\
\hline 1-month follow-up & $48(34.3)$ & $38(27.7)$ & $1.1(0.9,1.3)$ & & \\
\hline 3-month follow-up & $71(55.0)$ & $58(44.6)$ & $1.2(1.0,1.6)$ & & \\
\hline 6-month follow-up & $73(57.9)$ & $50(38.8)$ & $1.5(1.1,1.9)$ & & \\
\hline 12-month follow-up & $68(54.8)$ & $56(45.5)$ & $1.2(0.9,1.6)$ & & \\
\hline
\end{tabular}

${ }^{a}$ The number of subjects who were assessed at follow-up was 277 (140 CSM and 137 ASM) at 1 month, 259 (129 CSM and 130 ASM) at 3 months, 255 (126 CSM and 129 ASM) at 6 months, and 247 (124 CSM and 123 ASM) at 12 months

${ }^{b}$ Mixed effects model repeated measures (MMRM) analysis was used to compare group differences over 12 months, adjusting for age, time effect, and baseline value of outcome variable

${ }^{c}$ Defined as 3 or 4 (improved) on 0-4 scale. Repeated measures logistic modeling performed for this outcome

opioids were seldom initiated (1.4\% of CSM vs. $2.7 \%$ of ASM patients).

\section{Automated Monitoring and Self-Management}

At least 1 automated report was completed by $131 \mathrm{CSM}$ and 126 ASM patients, with a similar mean number of reports (14.5 vs. $14.0, P=.66$ ). CSM and ASM patients also completed a similar number of the 9 self-management modules (6.3 vs.
$5.8, P=.36)$. However, CSM patients were more likely to rate a greater proportion of their completed modules as moderately or very helpful $(65.6 \%$ vs. $52.5 \%, P<.001)$.

\section{Participant Satisfaction}

Satisfaction with pain and mood treatment was slightly but not significantly better in the CSM group (3.00 vs. 3.25 on a 3item satisfaction scale where $1=$ excellent and $5=$ poor,

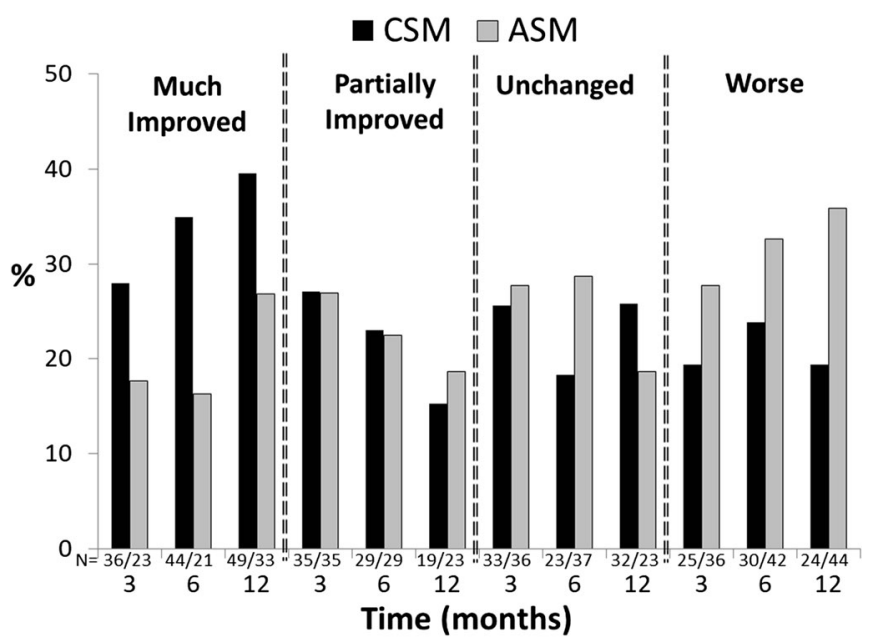

Fig. 2 Patient-reported global change in pain and mood. Patients rated their pain and mood separately as improved, unchanged, or worse at each follow-up assessment. They were classified as much improved if both pain and mood were improved; as partially improved if one of the symptoms (pain or mood) was improved, and the other symptom was unchanged; as unchanged if either both symptoms were unchanged, or one symptom was improved and the other symptom was worse; and worse if one symptom was worse, and the other symptom was worse or unchanged. The number of patients in the comprehensive symptom management (CSM) and assisted symptom management (ASM) arms who provided global change data was 129 and 130 at 3 months, 126 and 129 at 6 months, and 124 and 123 at 12 months. Between-group differences were not significant by chi-square analysis at 3 months $(P=.17)$, but were significant at 6 months $(P=.004)$ and 12 months $(P=.013)$. At 12 months, CSM patients were more likely than ASM patients to report being much improved (39.5\% vs. 26.8\%) and less likely to report worsening $(19.4 \%$ vs. $35.8 \%)$ 
Table 3 Secondary Symptom and Health-Related Quality of Life Outcomes in CAMMPS Trial Participants

\begin{tabular}{|c|c|c|c|c|c|}
\hline \multirow[t]{2}{*}{ Clinical outcome } & \multicolumn{2}{|l|}{ Mean (SD) } & \multirow{2}{*}{$\begin{array}{l}\text { Time-specific between-group } \\
\text { difference }(95 \% \mathrm{CI})\end{array}$} & \multirow{2}{*}{$\begin{array}{l}\text { Model estimate } \\
(95 \% \text { CI })^{b}\end{array}$} & \multirow{2}{*}{$\begin{array}{l}\text { Overall } P \\
\text { value }^{\mathrm{b}}\end{array}$} \\
\hline & CSM group (147) ${ }^{a}$ & $\operatorname{ASM}$ group $(147)^{\mathrm{a}}$ & & & \\
\hline \multicolumn{6}{|c|}{ BPI pain total (range, $0-10$ ) } \\
\hline Baseline & $6.0(1.7)$ & $5.8(1.7)$ & $0.2(-0.2,0.6)$ & \multirow{5}{*}{$-0.32(-0.65,0.004)$} & \multirow{5}{*}{.05} \\
\hline 1-month follow-up & $5.4(1.9)$ & $5.5(2.1)$ & $-0.1(-0.6,0.4)$ & & \\
\hline 3-month follow-up & $5.1(2.2)$ & $5.2(2.0)$ & $-0.2(-0.7,0.3)$ & & \\
\hline 6-month follow-up & $5.0(2.3)$ & $5.2(2.2)$ & $-0.2(-0.8,0.3)$ & & \\
\hline 12-month follow-up & $5.0(2.2)$ & $5.0(2.3)$ & $0.0(-0.6,0.5)$ & & \\
\hline \multicolumn{6}{|c|}{ GAD-7 anxiety (range, $0-21$ ) } \\
\hline Baseline & $11.3(5.0)$ & $11.1(5.3)$ & $0.2(-1.0,1.4)$ & $-0.82(-1.69,0.04)$ & .06 \\
\hline 1-month follow-up & $8.7(5.1)$ & $9.2(5.7)$ & $-0.4(-1.7,0.8)$ & & \\
\hline 3-month follow-up & $8.4(5.5)$ & $9.1(5.7)$ & $-0.7(-2.0,0.7)$ & & \\
\hline 6-month follow-up & $7.8(5.6)$ & $8.1(5.6)$ & $-0.3(-1.7,1.1)$ & & \\
\hline 12-month follow-up & $7.5(5.4)$ & $8.4(6.2)$ & $-0.9(-2.3,0.6)$ & & \\
\hline PHQ-9 depression (rang & $0-27)$ & & & & \\
\hline Baseline & $14.4(5.1)$ & $13.7(5.3)$ & $0.7(-0.4,1.9)$ & $-1.51(-2.42,-0.60)$ & .001 \\
\hline 1-month follow-up & $11.7(5.3)$ & $12.5(6.0)$ & $-0.7(-2.1,0.6)$ & & \\
\hline 3-month follow-up & $11.4(6.1)$ & $12.3(6.1)$ & $-0.9(-2.3,0.6)$ & & \\
\hline 6-month follow-up & $10.3(6.2)$ & $11.6(6.1)$ & $-1.3(-2.8,0.2)$ & & \\
\hline 12-month follow-up & $10.3(5.3)$ & $11.4(5.9)$ & $-1.2(-2.6,0.2)$ & & \\
\hline PROMIS fatigue T-score & lorm $=50)$ & & & & \\
\hline Baseline & $63.6(8.2)$ & $62.9(8.3)$ & $0.7(-1.2,2.6)$ & $-1.38(-2.99,0.22)$ & .09 \\
\hline 3-month follow-up & $60.7(8.8)$ & $61.5(9.6)$ & $-0.8(-3.1,1.5)$ & & \\
\hline 12-month follow-up & $60.3(9.6)$ & $60.6(9.9)$ & $-0.3(-2.8,2.1)$ & & \\
\hline PROMIS sleep T-score ( & $\mathrm{rm}=50)$ & & & & \\
\hline Baseline & $59.6(7.6)$ & $59.7(7.8)$ & $-0.1(-1.9,1.7)$ & $-0.88(-2.45,0.70)$ & .27 \\
\hline 3-month follow-up & $57.9(9.5)$ & $59.3(8.1)$ & $-1.4(-3.6,0.8)$ & & \\
\hline 12-month follow-up & $57.4(9.0)$ & $57.7(9.4)$ & $-0.3(-2.6,2.0)$ & & \\
\hline Pain catastrophizing, me & (SD) (range, 0-36) & & & & \\
\hline Baseline & $19.0(8.8)$ & $17.7(9.2)$ & $1.4(-0.7,3.4)$ & $-1.63(-3.34,0.08)$ & .06 \\
\hline 6-month follow-up & $14.4(9.8)$ & $15.0(9.6)$ & $-0.6(-3.0,1.8)$ & & \\
\hline 12-month follow-up & $13.4(9.7)$ & $13.7(10.1)$ & $-0.2(-2.7,2.2)$ & & \\
\hline REMIT depression remi & on score (range 0-20) & & & & \\
\hline Baseline & $9.1(3.8)$ & $8.9(3.8)$ & $0.2(-0.7,1.1)$ & $-0.61(-1.29,0.07)$ & .08 \\
\hline 3-month follow-up & $8.5(4.1)$ & $8.9(4.3)$ & $-0.4(-1.4,0.6)$ & & \\
\hline 6-month follow-up & $7.9(4.5)$ & $8.7(4.2)$ & $-0.8(-1.9,0.2)$ & & \\
\hline 12-month follow-up & $8.3(4.3)$ & $8.5(4.1)$ & $-0.3(-1.3,0.8)$ & & \\
\hline PTSD checklist (range, & $-85)$ & & & & \\
\hline Baseline & $44.1(14.7)$ & $45.6(15.4)$ & $-1.5(-5.0,1.9)$ & $-1.33(-4.20,1.54)$ & .36 \\
\hline 3-month follow-up & $39.9(17.6)$ & $41.1(15.7)$ & $-1.2(-5.3,2.9)$ & & \\
\hline 12-month follow-up & $39.2(18.0)$ & $41.5(21.0)$ & $-2.3(-7.2,2.6)$ & & \\
\hline PHQ-14 somatization (ra & e, $0-28)$ & & & & \\
\hline Baseline & $13.9(3.9)$ & $13.2(4.2)$ & $0.8(-0.2,1.7)$ & $-0.93(-1.77,-0.08)$ & .03 \\
\hline 12-month follow-up & $11.7(4.4)$ & $12.1(4.8)$ & $-0.4(-1.5,0.8)$ & & \\
\hline SF-12 (range, $0-100)$ & & & & & \\
\hline Physical component su & mary & & & & \\
\hline Baseline & $33.2(8.6)$ & $33.2(8.4)$ & $0.0(-1.9,1.9)$ & $1.19(-0.26,2.64)$ & .11 \\
\hline 3-month follow-up & $34.6(8.2)$ & $33.5(9.0)$ & $1.1(-1.0,3.2)$ & & \\
\hline 12-month follow-up & $33.9(8.7)$ & $33.4(9.4)$ & $0.5(-1.8,2.7)$ & & \\
\hline Mental component sun & ary & & & & \\
\hline Baseline & $37.5(11.2)$ & $38.2(10.1)$ & $-0.7(-3.1,1.8)$ & $1.28(-0.81,3.38)$ & .23 \\
\hline 3-month follow-up & $40.6(12.3)$ & $39.4(12.7)$ & $1.2(-1.9,4.2)$ & & \\
\hline 12-month follow-up & $41.8(12.5)$ & $41.4(12.5)$ & $0.5(-2.7,3.6)$ & & \\
\hline SF-36 (range, $0-100$ ) & & & & & \\
\hline Social functioning & & & & & \\
\hline Baseline & $43.3(26.1)$ & $43.6(26.6)$ & $-0.3(-6.4,5.7)$ & $6.21(1.35,11.06)$ & .01 \\
\hline 3-month follow-up & $55.9(28.3)$ & $49.0(29.2)$ & $6.9(-0.2,13.9)$ & & \\
\hline 12-month follow-up & $54.6(27.8)$ & $50.3(30.2)$ & $4.3(-2.9,11.6)$ & & \\
\hline Vitality & & & & & \\
\hline Baseline & $27.6(17.0)$ & $27.5(20.4)$ & $0.1(-4.2,4.4)$ & $3.50(0.29,6.70)$ & .03 \\
\hline 3-month follow-up & $32.1(19.8)$ & $28.6(20.9)$ & $3.5(-1.5,8.5)$ & & \\
\hline 12-month follow-up & $33.2(20.0)$ & $32.1(23.2)$ & $1.2(-4.3,6.6)$ & & \\
\hline Mental health & & & & & \\
\hline Baseline & $51.9(19.6)$ & $52.7(19.8)$ & $-0.8(-5.3,3.7)$ & $2.54(-1.06,6.13)$ & .17 \\
\hline 3-month follow-up & $57.1(21.4)$ & $55.0(22.3)$ & $2.1(-3.2,7.5)$ & & \\
\hline 12-month follow-up & $58.0(22.2)$ & $57.3(22.0)$ & $0.7(-4.8,6.2)$ & & \\
\hline Bodily pain & & & & & \\
\hline Baseline & $29.3(15.3)$ & $29.8(16.5)$ & $-0.5(-4.2,3.1)$ & $2.60(-0.90,6.11)$ & .14 \\
\hline 3-month follow-up & $35.2(18.1)$ & $33.6(18.0)$ & $1.6(-2.9,6.0)$ & & \\
\hline 12-month follow-up & $34.4(19.0)$ & $32.9(20.2)$ & $1.6(-3.3,6.5)$ & & \\
\hline General health percepti & & & & & \\
\hline Baseline & $37.9(27.0)$ & $37.8(29.0)$ & $0.1(-6.4,6.5)$ & $1.10(-3.17,5.36)$ & .61 \\
\hline 3-month follow-up & $37.4(26.9)$ & $36.6(28.7)$ & $0.8(-6.0,7.6)$ & & \\
\hline 12-month follow-up & $40.6(26.5)$ & $39.0(27.9)$ & $1.6(-5.2,8.4)$ & & \\
\hline
\end{tabular}


Table 3. (continued)

\begin{tabular}{|c|c|c|c|c|c|}
\hline \multirow[t]{2}{*}{ Clinical outcome } & \multicolumn{2}{|l|}{ Mean (SD) } & \multirow{2}{*}{$\begin{array}{l}\text { Time-specific between-group } \\
\text { difference }(95 \% \mathrm{CI})\end{array}$} & \multirow{2}{*}{$\begin{array}{l}\text { Model estimate } \\
(95 \% \text { CI })^{b}\end{array}$} & \multirow{2}{*}{$\begin{array}{l}\text { Overall } P \\
\text { value }^{b}\end{array}$} \\
\hline & CSM group $(147)^{a}$ & $\operatorname{ASM}$ group (147) ${ }^{\mathrm{a}}$ & & & \\
\hline \multicolumn{6}{|c|}{ Sheehan Disability Index (range, $0-10$ ) } \\
\hline Baseline & $5.7(2.3)$ & $5.7(2.4)$ & $0.0(-0.5,0.5)$ & $-0.43(-0.84,-0.02)$ & .04 \\
\hline 3-month follow-up & $4.9(2.6)$ & $5.3(2.6)$ & $-0.4(-1.0,0.2)$ & & \\
\hline 6-month follow-up & $4.8(2.7)$ & $5.2(2.7)$ & $-0.3(-1.0,0.3)$ & & \\
\hline 12-month follow-up & $5.0(2.5)$ & $5.4(2.8)$ & $-0.4(-1.1,0.3)$ & & \\
\hline \multicolumn{6}{|c|}{ Disability days 4 weeks, mean (SD) (range, $0-28$ ) } \\
\hline Baseline & $15.7(9.1)$ & $15.2(9.7)$ & $0.4(-1.7,2.6)$ & $1.09(0.94,1.27)$ & .24 \\
\hline 3-month follow-up & $13.7(8.9)$ & $13.5(9.8)$ & $0.2(-2.0,2.5)$ & & \\
\hline 6-month follow-up & $13.4(8.6)$ & $12.9(9.5)$ & $0.5(-1.8,2.7)$ & & \\
\hline 12 -month follow-up & $14.2(9.2)$ & $14.3(9.6)$ & $-0.1(-2.4,2.3)$ & & \\
\hline \multicolumn{6}{|c|}{$\%$ work effective, mean (SD) (range, $0-100)$} \\
\hline Baseline & $60.1(25.2)$ & $61.6(28.0)$ & $-1.5(-7.7,4.6)$ & $1.26(-3.14,5.67)$ & .57 \\
\hline 3-month follow-up & $60.9(25.0)$ & $60.8(24.5)$ & $0.1(-5.9,6.2)$ & & \\
\hline 6-month follow-up & $61.6(24.8)$ & $61.1(26.0)$ & $0.5(-5.7,6.8)$ & & \\
\hline 12-month follow-up & $62.0(25.8)$ & $59.2(27.8)$ & $2.8(-3.9,9.5)$ & & \\
\hline
\end{tabular}

${ }^{a}$ The number of subjects who were assessed at follow-up was 277 (140 CSM and 137 ASM) at 1 month, 259 (129 CSM and 130 ASM) at 3 months, 255 (126 CSM and 129 ASM) at 6 months, and 247 (124 CSM and 123 ASM) at 12 months

${ }^{b}$ Mixed effects model repeated measures (MMRM) analysis was used to compare group differences over 12 months, adjusting for age, time effect, and baseline value of outcome variable. Since PHQ-14 was measured only at baseline and 12 months, an ordinary regression model was used. Since disability days were count data, a Poisson regression with random effect model was used

$P=.07)$. Both groups found the automated reports easy $(74.7 \%)$ or only a little difficult $(19.8 \%)$ to complete. However, CSM patients were more likely to consider automated reporting very or moderately helpful $(64.8 \%$ vs. $46.5 \%$, $P=.006)$. CSM patients also rated the nurse care manager calls as very $(73.5 \%)$ or moderately $(16.8 \%)$ helpful.

\section{DISCUSSION}

The CAMMPS trial has several key findings. First, two telehealth-predominant strategies each resulted in moderate improvements in PAD symptoms over 12 months. Second, patient adherence and satisfaction were reasonably high, similar to 2 previous trials. ${ }^{25,26}$ Third, the intervention requiring clinical personnel resources (CSM) was superior to the exclusively automated intervention (ASM) by a small but clinically significant degree. Fourth, ASM patients were nearly twice as likely to report worsening at 12 months. The modest superiority of CSM may have been due to the combined effect of several small advantages of CSM compared to ASM including the use of more analgesics, greater perceived value of selfmanagement modules and automated monitoring, and the highly valued nurse contacts and care coordination.

The pain improvement demonstrated in CAMMPS for ASM (effect size of .52) exceeded improvement experienced by the usual care control group in 4 previous collaborative care trials for pain (effect size range across the 4 studies was .06 to $.38),{ }^{5-8}$ suggesting that ASM served as intended as an active comparator. As expected in a comparative effectiveness study, the between-group difference in CAMMPS (effect size of .23) was smaller than in 4 usual care control trials, where betweengroup effect sizes were $.27^{5}, .42^{7}, .57^{8}$, and $.77^{6}$. Also, cooccurring PAD symptoms can reduce treatment response of both pain and mood. ${ }^{27,28}$
One in five patients were receiving opioids at baseline, but this proportion decreased during the trial and few patients were initiated on opioids in either group. This might be due to nationwide efforts to reduce opioid use concurrent with our trial. Our stepped care approach across both groups, however, was consistent with prior effectiveness trials for chronic pain ${ }^{8}$, 29 and with opioid minimization guidelines ${ }^{30}$ and likely contributed.

This study addressed a common but difficult-to-treat patient population with persistent pain combined with at least moderately severe depression or anxiety. CAMMPS shows that an automated treatment approach (ASM) can be safely used but that CSM, with added human interaction through collaborative care, was comparatively more effective. Given these findings, one could opt for sequential administration, with web-based self-management and monitoring (ASM) initially followed by the more resource-intensive CSM for patients not responding.

Our study has limitations. CAMMPS was designed only to compare CSM to ASM and therefore cannot assess ASM effectiveness. In addition, the sample consisted of predominantly male veterans from a single medical center. Furthermore, VA nationally adopted primary care-mental health integration with care managers in 2007. ${ }^{31-33}$ While few patients, to our knowledge, were enrolled in CAMMPS and simultaneously followed by a non-CAMMPS care manager, the VA environment may differ from delivery systems without integrated mental health.

In summary, the CAMMPS trial supports expanded use of collaborative care-enhanced automated self-management among patients with PAD. Further research should assess effects of CSM on cost-effectiveness, access to effective care, and long-term outcomes for similarly complex chronic pain patients, as well as its implementation across various delivery system environments. 
Acknowledgments: Dr. Kroenke had full access to all the data in the study and takes responsibility for the integrity of the data and the accuracy of the data analysis. Fitsum Baye, Spencer Lourens, and Kurt Kroenke conducted and are responsible for the data analysis.

Corresponding Author: Kurt Kroenke, MD; Regenstrief Institute, Inc., Indianapolis, IN, USA (e-mail: kkroenke@regenstrief.org).

Funding This work was supported by a Department of Veterans Affairs Health Services Research and Development Merit Review award to Dr. Kroenke (IIR 12-095).

\section{Compliance with Ethical Standards:}

Conflict of Interest: The authors declare that they do not have a conflict of interest.

Disclaimer: The sponsor had no role in study design; in the collection, analysis, and interpretation of data; in the writing of the report; or in the decision to submit the article for publication. The views expressed in this article are those of the authors and do not necessarily represent the views of the Department of Veterans Affairs.

\section{REFERENCES}

1. Kroenke K, Evans E, Weitlauf S, et al. Comprehensive vs. Assisted Management of Mood and Pain Symptoms (CAMMPS) trial: Study design and sample characteristics. Contemp Clin Trials. 2018;64:179-87.

2. Institute of Medicine. Relieving Pain in America: A Blueprint for Transforming Prevention, Care, Education, and Research. Washington, DC: National Academies Press; 2011.

3. Kroenke $\mathbf{K}$, Unutzer $\mathbf{J}$. Closing the false divide: sustainable approaches to Integrating mental health services into primary care. J Gen Intern Med. 2017;32(4):404-10. https://doi.org/10.1007/s11606-016-3967-9

4. US Burden of Disease Collaborators. The state of US health, 1990-2010 burden of diseases, injuries, and risk factors. JAMA. 2013;310(6):591-608.

5. Dobscha SK, Corson K, Perrin NA, et al. Collaborative care for chronic pain in primary care: a clustered randomized trial. JAMA. 2009;301(12):1242-52.

6. Kroenke K, Bair MJ, Damush TM, et al. Optimized antidepressant therapy and pain self-management in primary care patients with depression and musculoskeletal pain: a randomized controlled trial. JAMA. 2009;301(20):2099-110.

7. Bair MJ, Ang D, Wu J, et al. Evaluation of Stepped Care for Chronic Pain (ESCAPE) in Veterans of the Iraq and Afghanistan conflicts: a randomized clinical trial. JAMA Intern Med 2015;175(5):682-9

8. Kroenke K, Krebs EE, Wu J, Yu Z, Chumbler NR, Bair MJ. Telecare collaborative management of chronic pain in primary care: a randomized clinical trial. JAMA. 2014;312(3):240-8.

9. Du S, Yuan C, Xiao X, Chu J, Giu Y, Qian H. Self-management programs for chronic musculoskeletal pain conditions: a systematic review and meta-analysis. Patient Educ Couns 2011;85(3):e299-310

10. Oliveira VC, Ferreira PH, Maher CG, Pinto RZ, Refshauge KM, Ferreira ML. Effectiveness of self-management of low back pain: systematic review with meta-analysis. Arthritis Care Res (Hoboken). 2012;64(11): 1739-48.

11. Heapy AA, Higgins DM, Cervone D, Wandner L, Fenton BT, Kerns RD A systematic review of technology-assisted self-management interventions for chronic pain: looking across treatment modalities. Clin J Pain 2015;31(6):470-92.

12. Boyers D, McNamee P, Clarke A, et al. Cost-effectiveness of selfmanagement methods for the treatment of chronic pain in an aging adult population: a systematic review of the literature. Clin J Pain. 2013;29(4):366-75.

13. Houle J, Gascon-Depatie M, Belanger-Dumontier G, Cardinal C. Depression self-management support: a systematic review. Patient Educ Couns. 2013;91(3):271-9.

14. Kroenke K, Spitzer RL, Williams JB, Lowe B. The Patient Health Questionnaire Somatic, Anxiety, and Depressive Symptom Scales: a systematic review. Gen Hosp Psychiatry 2010;32(4):345-59.
15. Krebs EE, Bair MJ, Wu J, Damush TM, Tu W, Kroenke K. Comparative responsiveness of pain outcome measures among primary care patients with musculoskeletal pain. Med Care. 2010;48:1007-14.

16. Kean $\mathbf{J}$, Monahan PO, Kroenke $\mathbf{K}$, et al. Comparative responsiveness of the PROMIS Pain Interference Short Forms, Brief Pain Inventory, PEG, and SF-36 Bodily Pain subscale. Med Care. 2016;54(4):414-21.

17. Von Ah D, Carpenter JS, Saykin A, et al. Advanced cognitive training for breast cancer survivors: a randomized controlled trial. Breast Cancer Res Treat. 2012;135(3):799-809.

18. Kazis LE, Anderson JJ, Meenan RF. Effect sizes for interpreting changes in health status. Med Care. 1989;27:S178-S89.

19. Cella D, Riley W, Stone A, et al. The Patient-Reported Outcomes Measurement Information System (PROMIS) developed and tested its first wave of adult self-reported health outcome item banks: 2005-2008. J Clin Epidemiol. 2010;63(11):1179-94.

20. Dube P, Kroenke K, Bair MJ, Theobald D, Williams LS. The P4 screener: evaluation of a brief measure for assessing potential suicidal risk in 2 randomized effectiveness trials of primary care and oncology patients. Prim. Care Companion. J Clin Psychiatry. 2010;12(6):e1-e8.

21. Kroenke K, Krebs EE, Bair MJ. Pharmacotherapy of chronic pain: a synthesis of recommendations from systematic reviews. Gen Hosp Psychiatry. 2009;31(3):206-19.

22. Kroenke $\mathbf{K}$, Theobald $\mathbf{D}$, Wu $\mathbf{J}$, et al. Effect of telecare management on pain and depression in patients with cancer: a randomized trial. JAMA. 2010;304(2): 163-71.

23. Mallinckrodt CH, Clark WS, David SR. Type I error rates from mixed effects model repeated measures versus fixed effects ANOVA with missing values imputed via last observation carried forward. Drug Inf $\mathrm{J}$. 2001;35:1215-25.

24. Little RJA. Modeling the drop-out mechanism in repeated-measures studies. J Am Stat Assoc. 1995;90:1112-21.

25. Johns SA, Kroenke $\mathbf{K}$, Theobald $\mathbf{D}, \mathbf{W u} \mathbf{J}$, Tu W. Telecare management of pain and depression in patients with cancer: patient satisfaction and predictors of use. J Ambul Care Manage 2011;34:126-39.

26. Guilkey RE, Draucker CB, Wu J, Yu Z, Kroenke $\mathbf{K}$. Acceptability of a telecare intervention for persistent musculoskeletal pain. J Telemed Telecare. 2018;24(1):44-50.

27. Kroenke K, Wu J, Bair MJ, Krebs EE, Damush TM, Tu W. Reciprocal relationship between pain and depression: a 12-month longitudinal analysis in primary care. J Pain. 2011;12:964-73.

28. Bair MJ, Poleshuck EL, Wu J, et al. Anxiety but not social stressors predict 12-month depression and pain outcomes. Clin J Pain. 2013;29(2):95-101

29. Krebs EE, Gravely A, Nugent S, et al. Effect of opioid vs nonopioid medications on pain-related function in patients with chronic back pain or hip or knee osteoarthritis Pain: the SPACE randomized clinical trial. JAMA. 2018;319(9):872-82.

30. Dowell D, Haegerich TM, Chou R. CDC Guideline for Prescribing Opioids for Chronic Pain-United States, 2016, JAMA. 2016;315(15):1624-45.

31. Rubenstein LV, Chaney EF, Ober S, et al. Using evidence-based quality improvement methods for translating depression collaborative care research into practice. Fam Syst Health. 2010;28(2):91-113

32. Chaney EF, Rubenstein LV, Liu CF, et al. Implementing collaborative care for depression treatment in primary care: a cluster randomized evaluation of a quality improvement practice redesign. Implement Sci 2011;6(1):121.

33. Whitebird RR, Solberg LI, Jaeckels NA, et al. Effective implementation of collaborative care for depression: what is needed? Am J Manag Care 2014;20(9):699-707

Publisher's Note Springer Nature remains neutral with regard to jurisdictional claims in published maps and institutional affiliations. 